\title{
喀斯特洞穴滴水细菌群落组成及其代谢功能 的季节性变化
}

云媛 1,2 , 程晓钰 ${ }^{1,2}$, 王纬琦 ${ }^{1,2}$, 王红梅 ${ }^{1,2 *}$

1. 中国地质大学(武汉)环境学院, 武汉 430074;

2. 中国地质大学(武汉), 生物地质与环境地质国家重点实验室, 武汉 430074

* 联系人, E-mail: hmwang@ @cug.edu.cn, wanghmei04@163.com

2018-06-26 收稿, 2018-09-25 修回, 2018-09-27 接受, 2018-10-30 网络版发表

国家自然科学基金重点项目(41130207)资助

摘要洞穴是研究地下生物圈的天然实验室, 而滴水是连接洞穴内部和外部环境的桥梁, 对滴水微生物群落结 构以及代谢功能进行研究, 可以为洞穴中微生物组成、来源以及微生物驱动的元素循环提供重要信息. 本研究采 用 Illumina Miseq 平台的 16S rRNA 二代测序技术及 Biolog-ECO 微平板检测法, 对湖北和尚洞弱光带滴水中的细 菌群落多样性、细菌群落代谢功能多样性以及环境因子进行了为期 2 年的动态监测. 结果表明: 洞穴滴水中优势 细菌属为不动杆菌属(Acinetobacter)和假单胞菌属(Pseudomonas)并表现出明显的季节性变化, 不动杆菌属 (Acinetobacter) 相对丰度在夏季最高、冬季最低，而假单胞菌属(Pseudomonas)相对丰度的季节性变化正好相反; 这 种季节性差异与宜昌地区的月平均降水量紧密相关. 同时, 基于 16S rRNA 的 PICRUSt 代谢功能预测及 Biolog-ECO 微平板法均显示洞穴滴水中细菌群落代谢功能具有季节性变化, 并且这种代谢能力的季节性变化与 不动杆菌属(Acinetobacter) 相对丰度的季节性变化显著正相关. 总之, 湖北宜昌和尚洞弱光区滴水中的细菌群落 结构的季节性变化与降水量密切相关, 同时优势类群不动杆菌属(Acinetobacter) 是滴水代谢能力的主要贡献者. 此研究将环境因子、滴水细菌群落组成及群落代谢能力三者紧密结合起来, 为进一步解译洞穴这一特殊的地下生 态系统中微生物群落组成及其代谢特征的变化规律奠定了基础.

关键词洞穴滴水, 细菌群落, 碳源代谢功能, 环境因子, 季节变化

洞穴是重要的陆地生态系统之一, 由于缺少阳 光、生物可用的有机碳和营养物质含量低, 加上地理 位置相对隔离等, 通常被认为是陆地黑暗生态系统中 的一种寡营养极端环境 ${ }^{[1]}$. 尽管如此, 洞穴中仍然生 存着大量的微生物 ${ }^{[25]}$, 使得洞穴成为研究地下生物圈 的天然实验室. 在洞穴生态系统中, 细菌、古菌和真菌 作为主要的生物类群广泛存在于洞穴各个小生境中, 包括风化岩壁 ${ }^{[6]}$ 、滴水 ${ }^{[7]}$ 、沉积物 ${ }^{[8]}$ 、上覆土壤 ${ }^{[9]}$ 、 石笋表面 ${ }^{[10]}$ 以及蝙蝠粪便 ${ }^{[11]}$ 等. 与真菌和古菌相比,
洞穴细菌研究相对较多. 洞穴中细菌主要类群为变形 菌门(Proteobacteria)、放线菌门(Actinobacteria)、厚壁 菌门(Firmicutes) 和拟杆菌门(Bacteroidetes), 且变形 菌门主要由 $\alpha-, \beta-, \gamma$-和 $\delta$-变形菌纲组成 ${ }^{[3,8,12]}$; 洞穴岩 壁与石笋表面细菌群落结构相似, 以变形菌门和放 线菌门为主 ${ }^{[3,8,12]}$; 洞穴沉积物与上覆土壤中的细菌 丰富度以及群落结构相似 ${ }^{[10,12]}$.

洞穴内有机质含量十分有限, 滴水作为连接洞 穴内部和外部环境的桥梁，在有机质进入洞穴的过

引用格式: 云媛, 程晓钰, 王纬琦, 等. 喀斯特洞穴滴水细菌群落组成及其代谢功能的季节性变化. 科学通报, 2018, 63: 3932-3944 Yun Y, Cheng X Y, Wang W Q, et al. Seasonal variation of bacterial community and their functional diversity in drip water from a karst cave (in Chinese). Chin Sci Bull, 2018, 63: 3932-3944, doi: 10.1360/N972018-00627 
程中起着举足轻重的作用 ${ }^{[13]}$. 对滴水微生物群落结 构以及代谢功能进行研究能为洞穴中微生物组成、来 源以及微生物驱动的元素循环提供重要信息. 目前 为止, 利用无机地球化学的方法对洞穴滴水的研究 已经取得重大进展 ${ }^{[14,15]}$, 但关于洞穴滴水微生物的 研究十分匮乏. 对洞穴滴水中微生物脂肪酸监测, 发 现冬季和春季滴水样本中的不饱和脂肪酸与饱和同 系物比值高于夏季 ${ }^{[16]}$. 运用传统的纯培养方法对滴 水微生物群落的研究 ${ }^{[7,17]}$ 显示, 可培养类群均为变形 菌门, 且以假单胞菌属(Pseudomonas)、丛毛单胞菌属 (Comamonas) 和不动杆菌属 (Acinetobacter) 为主. 利 用 $16 \mathrm{~S}$ rRNA克隆文库测序法, 对湖北清江和尚洞滴 水进行了为期 5 年的细菌群落组成的监测, 结果很好 地揭示了洞穴滴水细菌群落结构的季节性变化, 秋 冬季节与春夏季节分别以 $\gamma$-变形菌门和 $\beta$-变形菌门 为主, 且温度是与滴水细菌群落结构变化显著相关 的环境因子 ${ }^{[17]}$. 此外, 利用Biolog-ECO微平板对滴 水碳源活性的季节性变化进行了监测, 发现不同滴 点不同季节碳源代谢活性均不相同 ${ }^{[18]}$. 但目前人们 对洞穴滴水微生物群落结构的动态变化与其代谢功 能变化之间的关系尚不清楚, 且对群落结构和代谢 功能发生变化的影响因素也不明确. 因此, 为了探讨 上述问题, 本研究采用Illumina Miseq高通量测序平 台的16S rRNA二代测序技术及Biolog-ECO微平板检 测法同时对洞穴滴水的细菌群落结构和代谢特征进 行了为期 2 年的分析, 以期揭示微生物群落组成和生 态功能之间的相关性以及细菌群落变化与环境因子 间的关系, 并为进一步解译洞穴这一地下生态系统 中微生物群落的季节性动态特征及其潜在的生态功 能奠定基础.

\section{1 材料与方法}

\section{1 样品的采集}

和尚洞位于湖北省宜昌市长阳县的清江南岸, 地理位置为 $30^{\circ} 27^{\prime} \mathrm{N}, 110^{\circ} 25^{\prime} \mathrm{E}$, 属季风性山地湿润气 候 ${ }^{[19]}$. 和尚洞发育于寒武纪白云岩中, 洞内有季节 性地下河, 且常年有滴水, 生存有少量蝙蝠. 洞穴未 进行旅游开发, 平时除了中国地质大学(武汉)的科研 人员进行定期的监测活动外, 没有外来人员. 由于洞 内黑暗, 缺乏直接来自光合作用的有机质, 加上地理 位置相对隔离等, 属于典型的寡营养喀斯特洞穴. 从
2014年7月 2016年6月对洞穴中部弱光带的一处滴点 (DW3或4D) 的滴水进行了为期两年的监测取样, 具 体采样点位置信息参考Yun等人 ${ }^{[12]}$, 采样时间、季节 及样品编号信息见表1. 由于大气降水抵达和尚洞内 需要近一个月的时间 ${ }^{[19]}$, 因此滴水的季节与采样月 份的对应关系如下: 1 3月份为冬季, 4 6月份为春季, 7 9月份为夏季, 10 12月份为秋季. 采样时利用无菌 的玻璃漏斗和 $10 \mathrm{~L}$ 无菌塑料桶收集滴水, 并用秒表 记下每分钟滴水点的滴速. 分装部分滴水至 $250 \mathrm{~mL}$ 无菌玻璃瓶中, 置于 $4{ }^{\circ} \mathrm{C}$ 冰箱保存样品, 用于后续 Biolog-ECO微平板检测以及理化参数的测定. 剩余 滴水样本运回实验室后, 立即在超净台中进行抽滤, 将微生物细胞收集在 $0.22 \mu \mathrm{m}$ 滤膜 $(0.22 \mu \mathrm{m}, 47 \mathrm{~mm}$, Supor-200, Pall Corporation, USA)上，随后将富集有 微生物细胞滤膜装人 $50 \mathrm{~mL}$ 无菌离心管中, 并保存在 $-80^{\circ} \mathrm{C}$ 冰箱, 用于后续DNA的提取.

\section{2 理化参数及细胞数目的测定}

用便携式多参数水质分析仪 $(\mathrm{HQ} 30 \mathrm{D}, \mathrm{HACH}$, Loveland, CO, USA)现场测定滴水的 $\mathrm{pH}$ 、电导率(EC) 和温度; 利用离子色谱(ICS-600, Thermo, USA)测定 滴水阴离子 $\left(\mathrm{NO}_{3}^{-}\right.$和 $\left.\mathrm{SO}_{4}^{2-}\right)$ 和阳离子 $\left(\mathrm{Ca}^{2+}\right.$ 和 $\left.\mathrm{Mg}^{2+}\right)$ 的含 量; 总有机碳(TOC)用元素分析仪(Vario TOC cube, Elementar, Hanau, Germany)进行测定; 利用苂光光 谱仪(FluoroMax-4, HORIBA Jobin Yvon, Edison, NJ, USA)测定滴水样本中的可溶荧光有机质指数 ${ }^{[20]}$, 包 括苂光强度 $(\mathrm{FI})^{[21]}$ 和腐殖化指数 $(\mathrm{HIX})^{[22]}$. 滴水中的 总细胞数采用呋叮橙染色, 样品与呋啶橙染液的比 值为 $(1 \mathrm{~mL} / 333 \mu \mathrm{L})$; 活细胞数利用细菌LIVE/DEAD ${ }^{\circledR}$ 试剂盒(ThermoFisher, USA)进行染色, 样品与染料 的比值为 $(1 \mathrm{~mL} / 100 \mu \mathrm{L})$, 在 40 倍苂光显微镜(BX51, Olympus, Germany)下随机计数 20 个视野的菌数.

\subsection{DNA 提取与测序}

采用PowerWater ${ }^{\circledR}$ DNA Isolation Kit(MOBIO, USA)提取滤膜上细胞的总DNA，对 $16 \mathrm{~S}$ rRNA基因的 V4区片段进行PCR扩增, 引物序列为 $520 \mathrm{~F}\left(5^{\prime}\right.$-AYT GGGYDTAAAGNG-3') 和 802R(5'-TACNVGGGTAT CTAATCC- $\left.3^{\prime}\right)^{[23]}$. PCR 产物通过 $2 \%$ 琼脂糖凝胶电泳 检测, 采用AxyPrepDNA凝胶回收试剂盒(AXYGEN, USA) 进行回收, PCR 扩增回收产物荧光试剂用 Quant-iT PicoGreen dsDNA Assay Kit进行苂光定量, 
之后在上海派森诺生物公司的Illumina MiSeq PE300 平台进行测序.

\section{4 滴水细菌功能多样性测定}

采用Biolog-ECO微平板技术分析对滴水中细菌 代谢功能多样性. 将 $150 \mu \mathrm{L}$ 水样加人到已经预热到 $25^{\circ} \mathrm{C}$ 的Biolog-ECO微平板中, 每个样品对每一种碳 源的利用有 3 个重复. 将接种后的Biolog-ECO微平板 放在保湿的容器中, 并置于 $25^{\circ} \mathrm{C}$ 黑暗的恒温培养箱 中进行长达 $144 \mathrm{~h}$ 的培养, 每隔 $24 \mathrm{~h}$ 在Biolog MicroStation(Biotek Instruments Inc., USA) 对微平板进 行检测, 读取每孔的吸光度值. 选取测定波长 $590 \mathrm{~nm}$ 下的数据用于后续分析 ${ }^{[24]}$.

\section{5 数据分析方法}

细胞总数和活细胞数按照公式 $X=(N \times S) /\left(S_{\mathrm{f}} \times V\right)$ 计 算, 其中 $X$ 为滴水含菌数 $($ cells $/ \mathrm{mL}), N$ 是各视野平均 个数(个), $S$ 是滤膜面积 $\left(\mathrm{mm}^{2}\right), S_{\mathrm{f}}$ 是显微镜视野面积

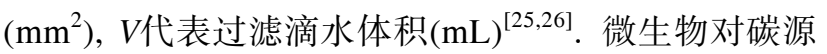
利用的多样性采用Biolog-ECO微平板中用来表征微 生物活性的重要指标——平均颜色变化率(AWCD)来 表示 ${ }^{[18,26,27]}$. 参考 Sala等人 ${ }^{[28]}$ 中的方法, 采用 Excel 2010 和SPSS 16.0软件对 $0 \sim 144 \mathrm{~h}$ 的数据进行积分和单 因素方差分析, 分别就微生物群落对Biolog-ECO微 平板中六大类碳源氨基酸、胺类、糖类、羧酸类、聚 合物类和芳香化合物类的利用情况进行分析.

利用QIIME软件(Version 1.9.1, http://qiime.org/) 对原始下机数据进行质控; 利用UCHIME ${ }^{[29]}$ 与 Gold database (http://drive5.com/uchime/uchime_download. html)数据库进行比对去除嵌合体, 从而得到优质序 列. 利用UCLUST的方法按照 $97 \%$ 的相似性将优质序 列聚类成可操作分类单元 $(\mathrm{OTU})^{[30]}$; 用RDP Classifier (Version 2.2, http://sourceforge.net/projects/rdp-classifier/)方法与GreenGene数据库 (http://greengenes.lbl. gov/cgi-bin/nph-index.cgi) 对OTU进行物种注释分析 (阈值为 0.8 ). 在深度为 33153 的条件下将所有样本的 序列进行均一化, 之后使用 QIIME软件 (Version 1.9.1)计算OTU数目, Chao1, Shannon, Simpson和ACE 指数. 采用PICRUSt将 $16 \mathrm{~S}$ rRNA基因序列在KEGG数 据库 (http://www.genome.jp/kegg/pathway.html) 中进 行功能预测分析 ${ }^{[31]}$; 细菌碳源利用率和PICRUSt功能 预测数据的箱型图在 R 软件上绘制. 细菌群落的主成
分分析(PCA)及其与环境因子、PICRUSt代谢功能和碳 源代谢能力的圥余分析 (RDA)均利用Canoco 5.0实 现 ${ }^{[32]}$, 其中在对细菌群落与环境因子的圥余分析中, 所有的环境参数均参与了分析, 但由于大部分环境 因子不显著, 图中只显示了能与前期结果 ${ }^{[17]}$ 进行对 比的环境因子.

\section{2 结果分析}

\section{1 洞穴滴水细菌群落结构特征}

洞穴滴水中的细菌以变形菌门(Proteobacteria, 平均相对丰度为 $82.26 \%$ ) 占主导, 尤其是 $\gamma$-变形菌纲 (Gammaproteobacteria), 其中不动杆菌属 (Acinetobacter) 和假单胞菌属 (Pseudomonas) 相对丰度最高, 且表现出明显的季节性变化. 不动杆菌属(Acinetobacter $)$ 的相对丰度在夏季最高, 冬季最低; 而假单胞 菌属(Pseudomonas)则与之相反(图1)。细菌丰度及多 样性指数在采样期间波动较大，没有显著的季节性 差异 $(P<0.05$, 表 1$)$. 例如, 反映细菌群落丰度的OTU 数、 $\mathrm{ACE}$ 指数和 Chao1指数的波动范围分别为 331 2580，534 3219和 494 2955; 反映菌群多样性的 Shannon 指数和 Simpson 指数的变化范围分别为 1.83 7.58和0.29 0.91; 此外, 微生物的活细胞数、总 细胞数、以及活细胞与总细胞的比值也没有季节性差 异(表1).

基于滴水细菌门类组成进行主成分分析显示细 菌群落的结构表现出季节性差异(图2(a)). 两个主轴 的解释率分别为 $62.15 \%$ 和 $16.09 \%$, 除了一个秋季样 本(14-11), 其余的秋冬季节样本均聚类在一起, 其群 落组成相似性相对较高, 具体表现为 $\gamma$-变形菌纲 (Gammaproteobacteria)的相对丰度较低, 其他门类相 对丰度较高; 而春夏季节的样本分布较散, 群落组成 与秋冬季节正好相反, 表现为 $\gamma$-变形菌纲 (Gammaproteobacteria)的相对丰度较高，其他门类的相对丰 度较低(图2(a)).

\section{2 洞穴滴水细菌代谢功能特征}

基于16S rRNA的PICRUSt代谢功能预测显示细 菌群落代谢功能以氨基酸代谢(10.24\% 11.46\%)和糖 类代谢 $(8.41 \%$ 9.65\%)为主(图3), 所有代谢类型的相 对丰度由高到低依次为氨基酸代谢 $>$ 糖类代谢 $>$ 能量 代谢 $>$ 外源物质降解和代谢 $>$ 脂类代谢 $>$ 酶因子和维 

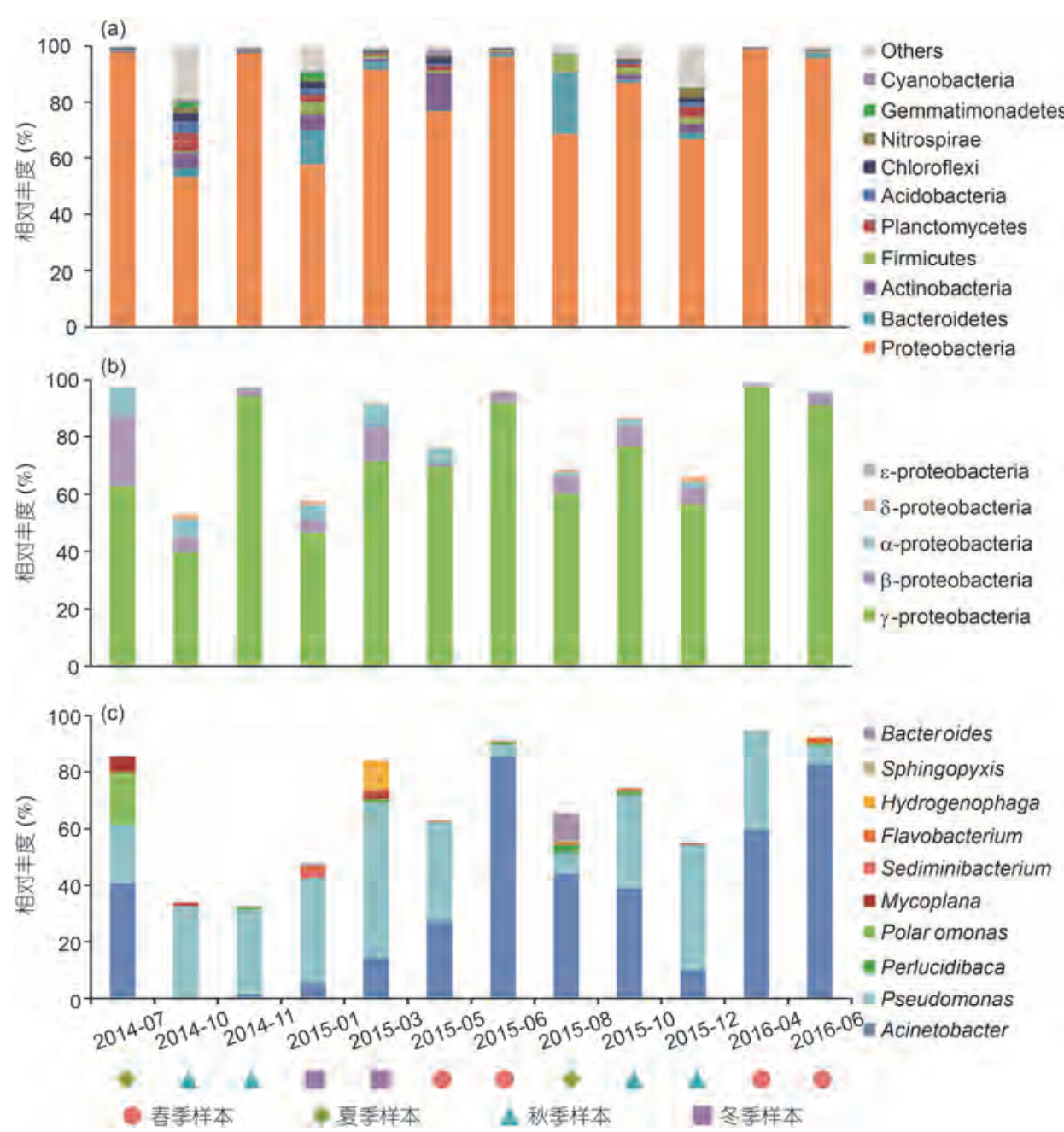

图 1 湖北清江和尚洞滴水中细菌群落结构. (a) 相对丰度排名前十的门; (b) 变形菌门; (c) 相对丰度排名前十的属

Figure 1 Bacterial community of the 10 most abundant phyla in the drip water at the phylum level (a), within Proteobacteria level (b), and at the genus level (c) in Heshang Cave

生素代谢 $>$ 萜类和聚酮化合物代谢 $>$ 多聚糖合成与代 谢 $>$ 酶家族, 整体代谢能力及外源物质降解和代谢具 有显著的季节性差异 $(P<0.05)$, 表现为春夏季节潜在 代谢功能丰度较高, 秋冬季节潜在代谢功能丰度较 低. 细菌群落对碳源利用的平均颜色变化率(AWCD) 也显示夏季(1.24 1.32)和春季(0.79 1.73)样本的细菌 活性最高, 其次为冬季(0.76 0.98)和秋季样本 $(0.38 \sim$ 0.76), 与活细胞和总细胞比值的变化一致(表 1 ). 细 菌群落对六类碳源的利用率结果显示细菌群落对胺 类和羧酸类的利用率较高, 且表现出显著的季节性 差异, 利用率的变化范围分别是 $0.29 \sim 2.52$ 和 $0.28 \sim$ 2.41 ; 其次为聚合物和糖类, 变化范围是 $0.33 \sim 2.47$ 和 0.49 2.11; 对芳香化合物和氨基酸类的利用率最低,
范围分别为 $0.1 \sim 1.3$ 和 $0.23 \sim 0.98$ (图 4). 此外, 基于滴 水细菌对六类碳源的利用率进行的PCA分析结果显 示两个主轴的解释率分别为 $59.63 \%$ 和 $21.86 \%$, 与基 于细菌门类的PCA结果一致(图2(b)): 秋冬季节的样 本聚类在一起, 整体表现出较低的碳源利用率; 而春 夏季节的样本则较为分散, 碳源利用率较高(图2(b)).

\section{3 洞穴滴水细菌群落与环境因子和代谢功能的 相关性}

洞穴外宜昌地区的月平均降水量、滴速、洞内外 温度及滴水电导率均表现出季节性的波动, 主要表 现为在5 8月份之间出现波峰，在11 12及 1 月份之间 出现波谷(图5); 同时温度(洞内和洞外)表现出显著 
表1 湖北清江和尚洞滴水中死活细胞数、细菌群落 OTU 数目、多样性指数、微生物利用碳源的平均颜色变化率 ${ }^{\text {a) }}$

Table 1 Summary of the bacterial cell number, operational taxonomic units (OTUs), diversity and average well color development (AWCD) value of drip waters in Heshang Cave, central China

\begin{tabular}{|c|c|c|c|c|c|c|c|c|c|c|c|}
\hline 季节 & 采样时间 & 编号 & $\begin{array}{l}\text { 总细胞数 } \\
\text { (cells/mL) }\end{array}$ & $\begin{array}{l}\text { 活细胞数 } \\
\text { (cells/mL) }\end{array}$ & $\begin{array}{c}\text { 活细胞/ } \\
\text { 总细胞 }(\%)\end{array}$ & OTUs & Shannon & Simpson & ACE & Chao1 & $\begin{array}{c}\text { 平均颜色变化 } \\
\text { 率(AWCD) }\end{array}$ \\
\hline \multirow{4}{*}{ 春季 } & 2015年05月 & $15-05$ & $2.03 \times 10^{5}$ & $3.67 \times 10^{4}$ & 18.09 & 999 & 4.98 & 0.86 & 1181.88 & 1171.07 & 0.79 \\
\hline & 2015年06月 & $15-06$ & $3.78 \times 10^{5}$ & $2.32 \times 10^{4}$ & 6.16 & 527 & 1.58 & 0.29 & 778.97 & 722.94 & 0.81 \\
\hline & 2016年04月 & $16-04$ & $2.66 \times 10^{4}$ & $7.75 \times 10^{3}$ & 29.17 & 331 & 1.83 & 0.56 & 534.65 & 494.38 & 1.73 \\
\hline & 2016年06月 & $16-06$ & - & - & - & 720 & 2.65 & 0.56 & 1494.46 & 1318.51 & 1.05 \\
\hline \multirow{2}{*}{ 夏季 } & 2014年07月 & $14-07$ & $7.77 \times 10^{4}$ & $2.72 \times 10^{4}$ & 35.04 & 1509 & 4.84 & 0.91 & 3218.65 & 2930.47 & 1.32 \\
\hline & 2015年08月 & $15-08$ & $3.02 \times 10^{5}$ & $1.13 \times 10^{4}$ & 3.73 & 1426 & 5.40 & 0.84 & 2318.41 & 2140.40 & 1.24 \\
\hline \multirow{4}{*}{ 秋季 } & 2014年10月 & $14-10$ & $1.07 \times 10^{5}$ & $4.98 \times 10^{3}$ & 4.67 & 2580 & 7.58 & 0.90 & 3013.07 & 2954.86 & 0.76 \\
\hline & 2014年11月 & $14-11$ & - & - & - & 480 & 2.15 & 0.57 & 686.62 & 631.23 & 0.59 \\
\hline & 2015年10月 & $15-10$ & $1.60 \times 10^{5}$ & $3.62 \times 10^{3}$ & 2.27 & 1142 & 4.21 & 0.77 & 1513.81 & 1535.50 & 0.38 \\
\hline & 2015年12月 & $15-12$ & - & - & - & 1771 & 5.92 & 0.82 & 2096.73 & 2064.49 & 0.61 \\
\hline \multirow{5}{*}{ 冬季 } & 2015年01月 & $15-01$ & - & - & - & 1476 & 6.50 & 0.87 & 1689.77 & 1681.83 & 0.76 \\
\hline & 2015年03月 & $15-03$ & $7.42 \times 10^{5}$ & $7.80 \times 10^{4}$ & 10.52 & 832 & 3.48 & 0.71 & 1318.10 & 1397.51 & 0.98 \\
\hline & $P$ 值(组间) & & 0.452 & 0.706 & 0.190 & 0.269 & 0.345 & 0.227 & 0.095 & 0.114 & 0.080 \\
\hline & $F$ & & 0.647 & 0.157 & 2.186 & 1.580 & 1.282 & 1.787 & 2.994 & 2.732 & 3.276 \\
\hline & $d f$ & & 1 & 1 & 1 & 3 & 3 & 3 & 3 & 3 & 3 \\
\hline
\end{tabular}

a) 表中的“-”代表未测数据
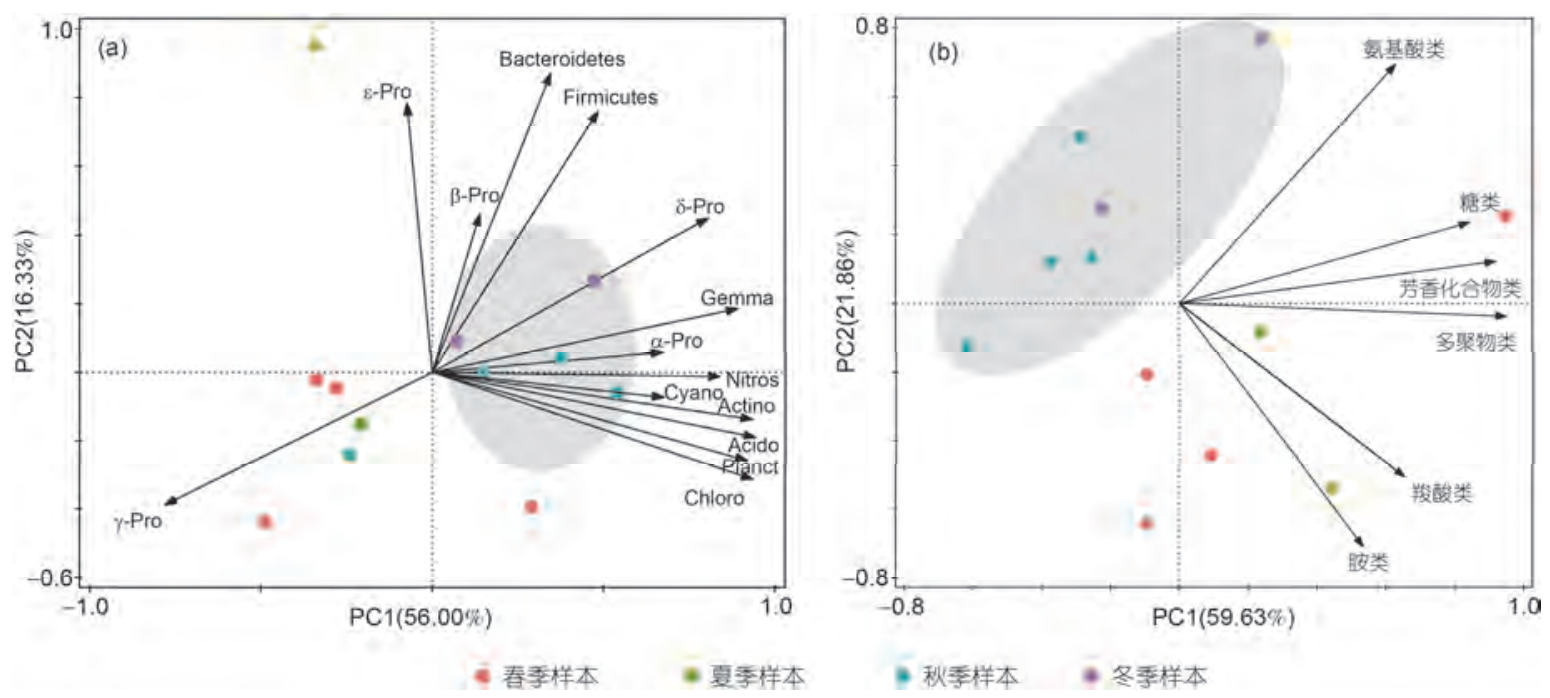

图 2 湖北清江和尚洞洞穴滴水样本细菌群落的主成分分析. (a) 基于门和变形菌门; (b) 基于微生物对六类碳源的利用率. 其中图中的简称 Pro, Gemma, Nitros, Cyano, Actino, Acido, Planct, Chloro, 分别代表变形菌门(Proteobacteria)、芽单胞菌门(Gemmatimonadetes)、硝化螺旋菌门 (Nitrospirae)、蓝细菌门(Cyanobacteria)、放线菌门(Actinobacteria)、酸杆菌门(Acidobacteria)、浮霉菌门(Planctomycetes)和绿弯菌门(Chloroflexi) Figure 2 Principal component analysis (PCA) of bacterial community according to taxon (a) and bacterial utilization of carbon sources based on Biolog-ECO plates in drip waters (b). The abbreviations in this figure were as follows: Pro, Proteobacteria; Gemma, Gemmatimonadetes; Nitros, Nitrospirae; Cyano, Cyanobacteria; Actino, Actinobacteria; Acido, Acidobacteria; Planct, Planctomycetes; Chloro, Chloroflexi

的季节性变化, $P$ 值分别为 0.011 和 0.032 (表 $S 1)$. 但是 洞穴滴水中的其他环境参数的季节性波动不明显(图 5 和表 $\mathrm{S} 1)$, 例如滴水 $\mathrm{pH}$ 波动无季节规律, 变化范围
为 7.76 8.53; $\mathrm{NO}_{3}^{-}, \mathrm{SO}_{4}^{2-}, \mathrm{Ca}^{2+}$ 和 $\mathrm{Mg}^{2+}$ 的浓度波动平缓, 浓度变化分别为 $13.83 \sim 16.39 \mathrm{mg} / \mathrm{L}, 26.04 \sim 32.26 \mathrm{mg} / \mathrm{L}$, $21.13 \sim 25.18 \mathrm{mg} / \mathrm{L}$ (除了一个异常值 $55.56 \mathrm{mg} / \mathrm{L}$ ) 和 


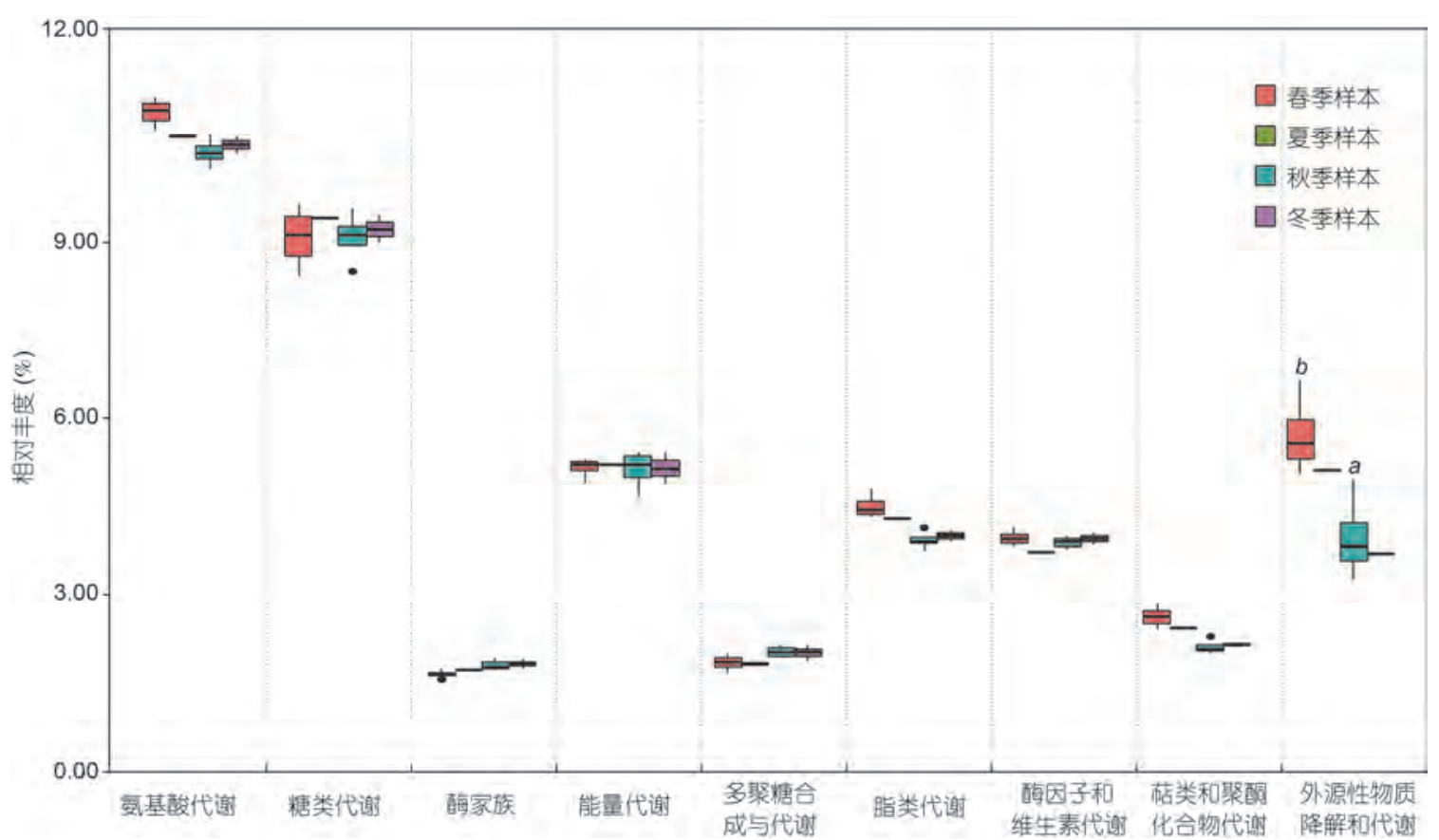

图 3 湖北清江和尚洞滴水中细菌群落潜在代谢类型的相对丰度. 其中, 数值 $a$ 和 $b$ 是指利用 SPSS 软件进行单因素方差分析后, 具有显著差 异 $(P<0.05)$ 的数值, 其余未标注的则代表组内数值均无显著差异

Figure 3 The relative abundance of potential metabolic types of bacterial communities based on 16S rRNA PICRUSt in drip waters. Based on one-way ANOVA (analysis of variance) calculations, different letters $(a, b)$ above the bars showed significantly difference $(P<0.05)$ among groups by using SPSS software, unlabeled letters represented no significant difference among groups

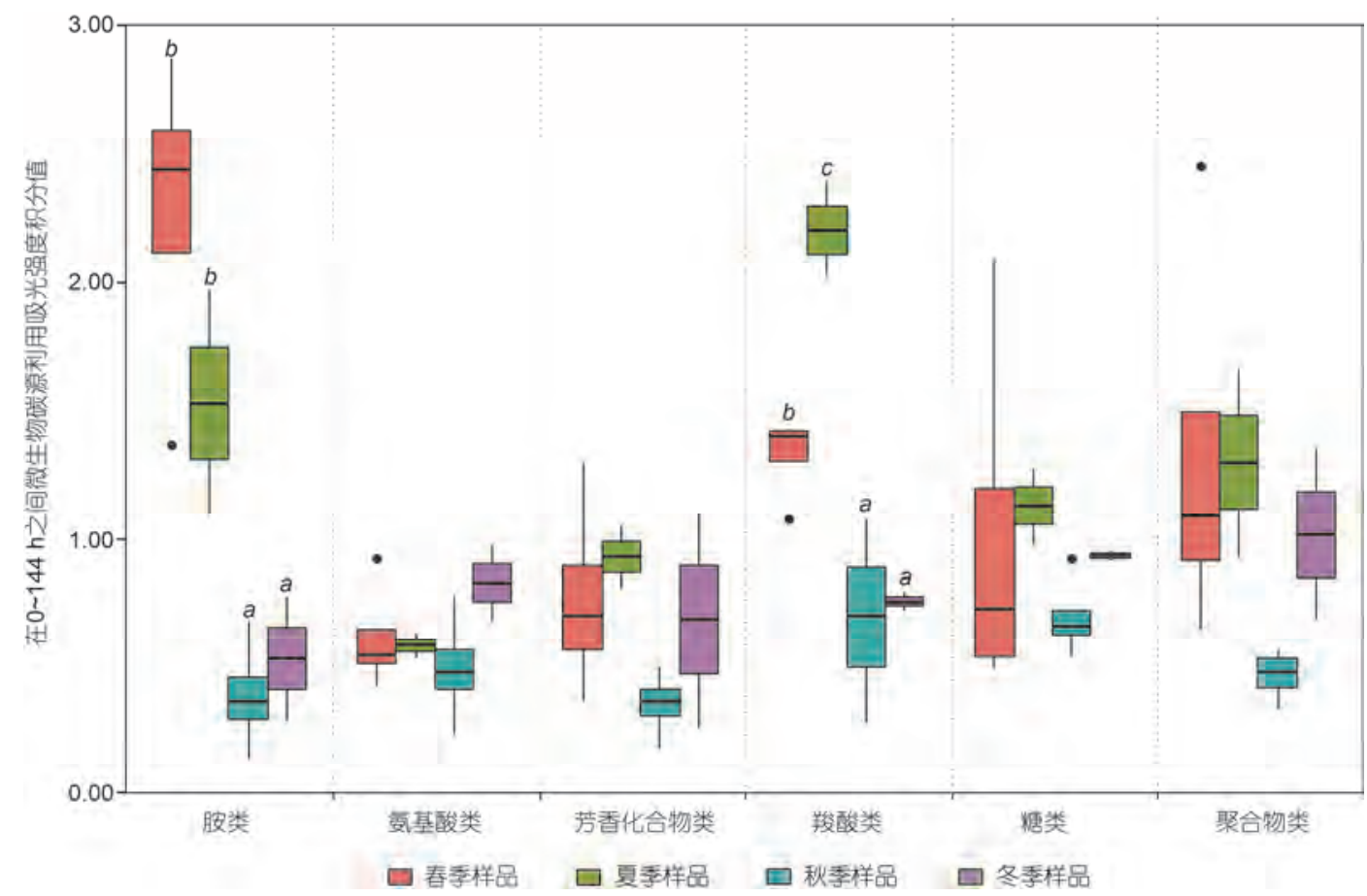

图 4 微生物对六类碳源利用率的箱型图. 其中, 数值 $a, b$ 和 $c$ 是指利用 SPSS 软件进行单因素方差分析后, 具有显著差异 $(P<0.05)$ 的数值, 其 余未标注的则代表组内数值无显著差异

Figure 4 Boxplots of bacterial utilization of carbon sources based on Biolog-ECO plates. Different letters $(a, b, c)$ above the bars showed significantly difference $(P<0.05)$ among different seasons, unlabeled letters represented no significant difference among groups 


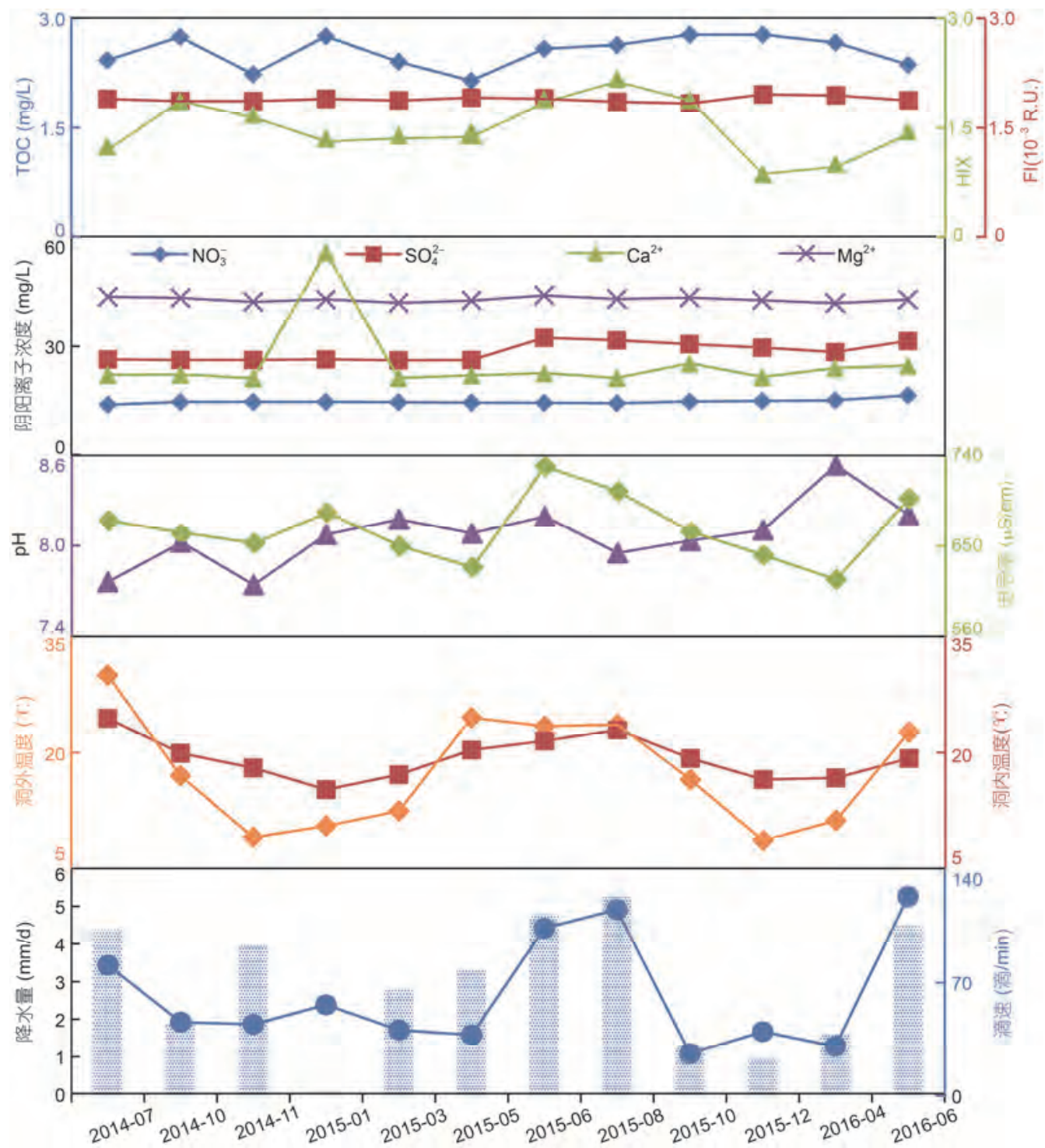

图 5 洞穴滴水中的环境参数信息, 其中 FI 为苂光指数, 代表样本中可溶有机质苂光强度; HIX 为腐殖化指数, 代表样本有机质腐殖化强度 Figure 5 Information of environmental parameters in drip waters. FI and HIX represent fluorescence index and humification index respectively

$41.71 \sim 43.82 \mathrm{mg} / \mathrm{L}$; 总有机质(TOC)含量、菼光有机质 强度 $(\mathrm{FI})$ 和腐殖化指数 $(\mathrm{HIX})$ 无季节性变化, 波动也 相对较平缓, 范围分别为 $2.14 \%$ 2.77\%, $1.83 \times 10^{-3}$ $1.95 \times 10^{-3}$ R.U.和0.86 2.17(图5和表S1).

细菌群落组成与环境因子的圥余分析 (RDA) 结 果(图6(a))显示, 第一轴和第二轴对细菌群落相对丰 度的解释度分别为 $56.00 \%$ 和 $16.33 \%$; 其中, 只有宜 昌地区的降水与细菌群落结构季节性变化显著相关 $(P=0.024)$, 并且降水量对细菌群落结构季节性变化 的解释度最高(27.6\%)(图6(b)). 同时在众多环境因子 中, 降水与洞内滴水滴速和洞内外温度显著正相关,
$R$ 值分别为 $0.734 * *, 0.758 * *$ 和 $0.693 *($ 图6(e)). 降水和 滴速与细菌群落优势属均有关系, 例如, 不动杆菌属 的相对丰度与滴水滴速的变化显著正相关 $(R=0.636$, $P<0.05)$; 而假单胞菌属的相对丰度与降水量 $(P<$ $0.01)$ 和滴速 $(P<0.001)$ 呈极显著的负相关关系(图 $6(\mathrm{e}))$. 此外, 与降水显著相关的洞内外温度只表现出 与假单胞菌属的显著负相关关系, $R$ 值分别为 $-0.665^{*}$ 和 $-0.667 *$ (图6(e)). 基于 $16 \mathrm{~S}$ rRNA的PICRUSt代谢能 力与细菌群落结构的RDA(图6(c))显示, 第一轴和第 二轴对细菌代谢能力的解释度分别为 $81.80 \%$ 和 $12.81 \%$, 而这种代谢能力的季节性差异主要与不动 



图 6 洞穴滴水中细菌群落结构与环境因子和代谢功能分析. (a) 群落结构与环境因子的冗余分析, 图中的简称与图 2 中的一致; (b) 环境因子 对群落结构变化的解释度; 基于 $16 \mathrm{~S}$ rRNA 的 PICRUSt 预测的代谢功能(c)和 Biolog-ECO 板碳源代谢能力(d)与群落结构的圥余分析; (e) 优势 属与环境因子及代谢功能相关性分析. 图中*,**和***分别代表 $P<0.05, P<0.01$ 和 $P<0.001$

Figure 6 The relationship between bacterial community and environmental factors and metabolic functions. (a) Redundancy analysis (RDA) of bacterial community and environmental factors; (b) the explanation of environmental factors to bacterial similarity pattern; redundancy analysis (RDA) of bacterial community between bacterial community and metabolic function based on 16S rRNA PICRUSt (c) and Biolog-ECO plates (d); correlation analysis among environmental factors, bacterial community and metabolic function (e). $* P<0.05$, ** $P<0.01$, and $* * * P<0.001$ 
杆菌属的相对丰度相关. 同时, 基于细菌碳源代谢能 力与细菌群落结构的圥余分析(RDA)表明(图6(d)), 第一轴和第二轴对细菌碳源代谢能力的解释度分别 为 $63.12 \%$ 和 $17.95 \%$, 其中不动杆菌属与细菌碳源代 谢显著相关, 与基于 $16 \mathrm{~S}$ rRNA的PICRUSt代谢能力 的结果一致. 此外, 优势属不动杆菌属的相对丰度与 基于PICRUSt预测的氨基酸代谢 $(P=0.012)$ 、脂类代谢 $(P<0.001)$ 、萜类和聚酮化合物代谢 $(P<0.001)$ 和外源 性物质降解和代谢 $(P<0.001)$, 以及与基于 Biolog$\mathrm{ECO}$ 微平板的胺类代谢 $(P=0.024)$ 均呈现显著正相关; 而优势属假单胞菌属与脂类代谢 $(P=0.030)$ 、萜类和 聚酮化合物代谢 $(P=0.009)$ 、外源性物质降解和代谢 $(P=0.009)$ 和羧酸代谢 $(P=0.025)$ 呈显著负相关(图6(e)).

\section{3 讨论}

\section{1 洞穴滴水细菌群落结构季节性变化及其与环 境因子的相关性}

洞穴滴水中的细菌群落以变形菌门(Proteobacteria)占主导, 且细菌群落的季节性变化主要与降水 量有关. 变形菌门普遍存在于陆地生态系统的水环 境中, 据报道在北海湖冬季水样和美国北卡罗纳州3 条河流的沉积物样品中变形菌门均是第一优势门 ${ }^{[33]}$, 且如果该门类的含量高于 $60 \%$ 时则暗示了环境中的 寡营养条件, 这些均与本研究的结果(变形菌门平均 相对丰度为 $82.26 \%$ ) 和洞穴滴水的寡营养条件相吻 合. 同时, 这些水环境中的细菌群落也具有季节性差 异, 如北海湖水样细菌群落中优势门在不同季节的 分布呈现季节性变化, 夏季和秋季样本中的细菌群 落以蓝细菌门(Cyanobacteria)为优势门, 冬季以变形 菌门(Proteobacteria)为主, 春季放线菌门(Actinobacteria)和拟杆菌门(Bacteroidetes)的丰度较高 ${ }^{[33]}$. 在美 国北卡罗纳州3条河流沉积物中的细菌群落结构也具 有季节性变化, 夏季样本中 $\gamma$-变形菌纲 (Gammaproteobacteria)的平均相对含量显著高于秋冬季节 ${ }^{[34]}$. 在本研究的洞穴滴水中, 细菌群落也呈现出季节性 变化, 并且春夏季节和秋冬季节样本的聚类情况与 基于克隆文库的研究结果一致, 但是 $\gamma$-变形菌纲 (Gammaproteobacteria), 尤其是不动杆菌属 (Acinetobacteria) 相对丰度在不同季节中的变化趋势却相 反 ${ }^{[17]}$, 原因可能是由于不同滴点水文动力学机制的 差异进而导致微生物群落对气候因子的差异响应.
基于克隆文库技术对和尚洞 $1 \mathrm{D}$ 和 $3 \mathrm{D}$ 两个滴点的细菌 群落结构进行了 5年(2008 2013年)的研究, 发现它们 的季节性变化主要与温度变化显著相关, 温度对细 菌群落季节性变化的解释度高达 $47 \%{ }^{[17]}$, 而本文中 的样本信息为 2 年(2014 2016年), 细菌群落信息与温 度变化的相关性相对较弱(解释度仅为 $6.6 \%$ ), 主要受 降水量的影响(解释度为 27.6\%)(图6(b)). 这种差异可 能是由于不同时间尺度降水量波动范围不同和不同 滴点滴速差异较大所造成, 2008 2013年降水量的波 动范围为 $0.22 \sim 4.43 \mathrm{~mm} / \mathrm{d}, 2014 \sim 2016$ 年间的降水量 波动为 $0.05 ~ 5.25 \mathrm{~mm} / \mathrm{d}$; 在春夏季节本文中滴点(4D) 的平均滴速 $(80$ 滴 $/ \mathrm{min})$ 高于 $1 \mathrm{D}(16$ 滴 $/ \mathrm{min})$ 和 $3 \mathrm{D}$ 的平均 滴速 $(57$ 滴/min), 秋冬季节的滴速也同样如此(40滴/ $\min$ 高于 32 滴 $/ \mathrm{min}$ 和 14 滴 $/ \mathrm{min})^{[17]}$. 水流量的增加会影 响洞穴上方非饱和带含水层, 进而对亲水性和疏水 性不同的微生物形成一定的选择作用, 从而形成了 微生物群落组成的差异 ${ }^{[35]}$. 本研究结果显示不动杆 菌属 (Acinetobacter) 与降水和滴速显著正相关 $(P<0.05)$, 降水多且滴速快的条件下 (湿润)不动杆菌 属(Acinetobacter)的相对含量较高, 且已有研究表明 不动杆菌属(Acinetobacter) 因其具有种间转化的遗传 学特征而经常在较湿润的环境中被发现, 相对于其 他细菌在高湿度的环境中具有更长的存活时间 ${ }^{[36 ~ 40]}$. 因此在今后的研究中, 应加强对洞穴水文动力学机 制的研究, 以期获得更加准确的微生物群落与环境 因子之间相互作用的信息.

\section{2 洞穴滴水细菌代谢能力季节性变化及其与细 菌群落结构的相关性}

基于 $16 \mathrm{~S}$ rRNA 的 PICRUSt 代谢功能预测和 Biolog-ECO微平板碳源利用率的这两种方法均显示 洞穴滴水中细菌群落代谢功能的季节性变化, 表现 为春夏季节代谢较高, 秋冬季节代谢缓慢. 这一现象 在很多生境中均有报道, 例如, 在对我国东北土壤微 生物群落代谢能力的季节性研究中发现土壤微生物 在夏季对碳源消耗量最大、代谢活性强, 秋季消耗量 最小且速率最低 ${ }^{[41]}$. 在水库的沉积物与水体交界面 同样发现细菌对碳源的利用效率具有季节性变化, 尤其表现为对糖类、氨基酸类和羧酸类的季节性代谢 明显 ${ }^{[42]}$. 同时, 已有研究表明微生物功能基因多样 性与微生物分类多样性密切相关, 功能多样性与群 落组成和结构多样性均具有很强的正相关关系 ${ }^{[43]}$. 
其中, 已经被报道的一些特定的关系有： $\gamma$-变形菌纲 (Gammaproteobacteria) 的相对丰度与亮氨酸氨基肽 酶 $(P<0.01)$ 和酚氧化酶 $(P<0.05)$ 代谢关系显著; $\beta$-变 形菌纲的相对丰度与葡糖苷酶 $(P<0.05)$ 、木糖苷酶 $(P<0.05)$ 和亮氨酸氨基肽酶 $(P<0.01)$ 的代谢显著相关; $\delta$-变形菌纲的相对丰度也与亮氨酸氨基肽酶的代谢 呈现出很强的正相关关系 $(P<0.05)^{[44]}$. 洞穴滴水的结 果显示, 细菌代谢功能的季节性变化与不动杆菌属 (Acinetobacter) 显著正相关, 尤其是氨基酸类代谢、 脂类代谢、萜类和聚酮化合物代谢、外源性物质降解 和代谢以及胺类代谢. 不动杆菌属 (Acinetobacter) 的 相对丰度与滴水整体细菌群落代谢能力的强弱如此 相关, 很有可能是由于不动杆菌属 (Acinetobacter)这 类细菌具有广谱性代谢能力 ${ }^{[37,39]}$. 例如, 比较蛋白质 组学和功能分析表明, A.baumannii的细胞表面具有 多种蛋白 (特别是尿刊酸酶) 参与组氨酸代谢并发挥 着重要作用 ${ }^{[45]}$; 同时利用蛋白学方法在A.baylyi 中发 现的一个小型非编码RNA(sRNA)-Aar被证实是氨基 酸代谢中的调控器 ${ }^{[46]}$. 在印度吉尔卡湖中分离的一 株A.sp. 的全基因组结果显示该菌株具有脂类代谢的 功能 ${ }^{[47]}$.

关于两种方法呈现出的主要代谢类型不一致的 现象, 一方面可能是由于Biolog-ECO微平板只能通 过31种单一有机碳源来评估异养微生物的代谢多样 性, 不能精确地表征洞穴滴水有机物底物的复杂性, 还会在一定程度上低估细菌群落的实际代谢水 平 ${ }^{[48,49]}$; 并且Biolog-ECO微平板的颜色变化主要取 决于异养细菌的丰度, 尤其是生长速率较高的菌群, 表征的只是细菌群落中可培养的、且具有代谢活性的 那部分细菌. 洞穴滴水本来是一个寡营养环境, 在
Biolog-ECO微平板这个富营养的条件下, 可能会对 原本在寡营养条件下具有较高代谢的类群产生一定 的抑制作用, 进而表现出细菌的代谢功能存在一定 差异 ${ }^{[50]}$. 另一方面, 基于 $16 \mathrm{~S}$ rRNA高通量测序进行 的PICRUSt代谢功能预测只是在DNA水平上预测细 菌群落具有某些代谢潜力, 但与群落实际能执行的 代谢功能之间也存在一定的差异. 但是, 将PICRUSt 代谢功能预测与Biolog-ECO微平板碳源代谢分析相 结合, 能够为我们提供更加完整的微生物群落代谢 功能的信息. 当然, 如果能结合DNA-SIP, 转录组和 蛋白组方面的研究, 则会为阐述微生物群落的代谢 功能提供更加完整和详尽的信息. 但环境样品十分 复杂, 代谢功能多种多样, 在实际研究中, 还要结合 具体的研究目的, 选择适当的技术和方法加以实施.

\section{4 结论}

湖北宜昌和尚洞弱光区滴水中的细菌主要以 $\gamma-$ 变形菌纲(Gammaproteobacteria)占主导, 春夏季节样 本中活细胞与总细胞的比值及细菌群落结构 (Acinetobacter 和Pseudomonas) 表现出季节性差异, 并且这种差异与宜昌地区的月平均降水量紧密相关. 同时, PICRUSt代谢功能预测和Biolog-ECO微平板碳 源利用率的这两种方法均显示洞穴滴水中细菌群落 代谢功能具有季节性变化, 并且这种代谢能力的季 节性变化与不动杆菌属(Acinetobacter)相对丰度的季 节性变化显著正相关. 此研究将环境因子与洞穴滴 水中微生物群落及代谢能力的联系起来, 为进一步 阐述深部生物圈中微生物驱动的元素循环、探讨洞穴 滴水微生物群落组成和代谢功能的时空变化特征奠 定了基础.

\section{参考文献}

1 Danielopol D L, Pospisil P, Rouch R. Biodiversity in groundwater: A large-scale view. Trends Ecol Evol, 2000, 15: 223-224

2 Engel A S. Microbial diversity of cave ecosystems. In: Geomicrobiology: Molecular and Environmental Perspective. Dordrecht: Springer, 2010. 219-238

3 Barton H A, Jurado V. What's up down there? Microbial diversity in caves. Microbe-AmSoc Microbiol, 2007, 2: 132

4 Barton H A, Giarrizzo J G, Suarez P, et al. Microbial diversity in a Venezuelan orthoquartzite cave is dominated by the Chloroflexi (class Ktedonobacterales) and Thaumarchaeota Group I.1c. FrontMicrobiol, 2014, 5: 615

5 Engel A S, Jones D S, Lavoie K H, et al. Microbial Life of Cave Systems. Berlin/Boston: De Gruyter, 2015

6 Ortiz M, Legatzki A, Neilson J W, et al. Making a living while starving in the dark: Metagenomic insights into the energy dynamics of a carbonate cave. ISME J, 2014, 8: 478-491

7 Liu Q, Wang H, Zhao R, et al. Bacteria isolated from dripping water in the oligotrophic Heshang cave in central China. J Earth Sci, 2010, 21: $325-328$ 
8 Wu Y, Tan L, Liu W, et al. Profiling bacterial diversity in a limestone cave of the western Loess Plateau of China. Front Microbiol, 2015, 6: 244

9 Ward N L, Challacombe J F, Janssen P H, et al. Three genomes from the phylum Acidobacteria provide insight into the lifestyles of these microorganisms in soils. Appl Environ Microbiol, 2009, 75: 2046-2056

10 Ortiz M, Neilson J W, Nelson W M, et al. Profiling bacterial diversity and taxonomic composition on speleothem surfaces in Kartchner Caverns, AZ. Microb Ecol, 2013, 65: 371-383

11 Man B, Wang H, Xiang X, et al. Phylogenetic diversity of culturable fungi in the Heshang cave, central china. Front Microbiol, 2015, 6: 1158

12 Yun Y, Wang H, Man B, et al. The relationship between $\mathrm{pH}$ and bacterial communities in a single karst ecosystem and its implication for soil acidification. Front Microbiol, 2016, 7: 1955

13 Lambert W J, Aharon P. Oxygen and hydrogen isotopes of rainfall and dripwater at desoto caverns (Alabama, USA): Key to understanding past variability of moisture transport from the gulf of Mexico. Geochim Cosmochim Acta, 2010, 74: 846-861

14 Tremaine D M, Froelich P N. Speleothem trace element signatures: A hydrologic geochemical study of modern cave dripwaters and farmed calcite. Geochim Cosmochim Acta, 2013, 121: 522-545

15 Baldini J U L, McDermott F, Baldini L M, et al. Identifying short-term and seasonal trends in cave drip water trace element concentrations based on a daily-scale automatically collected drip water dataset. Chem Geol, 2012, 330: 1-16

16 Li X, Wang C, Huang J, et al. Seasonal variation of fatty acids from drip water in Heshang cave, central China. Appl Geochem, 2011, 26: 341-347

17 Yun Y, Xiang X, Wang H, et al. Five-year monitoring of bacterial communities in dripping water from the Heshang cave in central China: Implication for paleoclimate reconstruction and ecological functions. Geomicrobiol J, 2016, 33: 1-11

18 Zhao R, Wang H M, Liu Q Y, et al. Spatial and seasonal variations of microbial communities in dripping water from the Heshang Cave, central China (in Chinese). Earth Sci-J China Univ Geosci, 2010, 35: 899-907 [赵锐, 王红梅, 刘倩瑛, 等. 湖北清江和尚洞滴水微生 物群落随空间和季节的变化. 地球科学: 中国地质大学学报, 2010, 35: 899-907]

19 Hu C, Henderson G M, Huang J, et al. Report of a three-year monitoring programme at Heshang cave, central China. Int J Speleol, 2008, 37: $143-151$

20 Brannen-Donnelly K, Engel A. Bacterial diversity differences along an epigenic cave stream reveal evidence of community dynamics, succession, and stability. Front Microbiol, 2015, 6: 729

21 McKnight D M, Boyer E W, Westerhoff P K, et al. Spectrofluorometric characterization of dissolved organic matter for indication of precursor organic material and aromaticity. Limnol Oceanogr, 2001, 46: 38-48

22 Ohno T. Fluorescence inner-filtering correction for determining the humification index of dissolved organic matter. Environ Sci Technol, 2002, 36: 742-746

23 Claesson M J, O'Sullivan O, Wang Q, et al. Comparative analysis of pyrosequencing and a phylogenetic microarray for exploring microbial community structures in the human distal intestine. PLoS One, 2009, 4: e6669

24 Jiang Z, Li P, Wang Y, et al. Effects of roxarsone on the functional diversity of soil microbial community. Int Biodeter Biodegr, 2013, 76: $32-35$

25 Hobbie J E, Daley R J, Jasper S. Use of nuclepore filters for counting bacteria by fluorescence microscopy. Appl Environ Microbiol, 1977, 33: 1225

26 Dong Q. The disstribution of phosphate solubilizing bacteria and the microbial response to environmental changes in Heshang Cave, Hubei (in Chinese). Doctor Dissertation. Wuhan: China University Geosciences, 2013 [董强. 湖北清江和尚洞洞穴系统溶磷微生物的分布 特征及滴水微生物对环境因子的响应. 博士学位论文. 武汉: 中国地质大学, 2013]

27 Meier A, Singh M K, Kastner A, et al. Microbial communities in carbonate rocks-from soil via groundwater to rocks. J Basic Microb, 2017, 57: 752-761

28 Sala M M, Pinhassi J, Gasol J M. Estimation of bacterial use of dissolved organic nitrogen compounds in aquatic ecosystems using biolog plates. Aquat Microb Ecol, 2006, 42: 1-5

29 Edgar R C, Haas B J, Clemente J C, et al. Uchime improves sensitivity and speed of chimera detection. Bioinformatics, 2011, 27: 2194-2200

30 Edgar R C. Search and clustering orders of magnitude faster than blast. Bioinformatics, 2010, 26: 2460-2461

31 Langille M G I, Zaneveld J, Caporaso J G, et al. Predictive functional profiling of microbial communities using 16s rRNA marker gene sequences. Nat Biotechnol, 2013, 31: 814

32 ter Braak C J F, Smilauer P. Canoco Reference Manual and Canodraw for Windows User'S Guide: Software for Canonical Community Ordination (Version 4.5). Ithaca, NY: Microcomputer Power, 2002

33 Zhang Y J, Li K, Zhu H R, et al. Community structure of microorganisms and its seasonal variation in Beihai lake (in Chinese). Environ Sci, 2017, 38: 3319-3329 [张雅洁, 李珂, 朱浩然, 等. 北海湖微生物群落结构随季节变化特征. 环境科学, 2017, 38: 3319-3329] 
34 Bucci J, Szempruch A, Caldwell J, et al. Seasonal changes in microbial community structure in freshwater stream sediment in a north Carolina river basin. Diversity, 2014, 6: 18

35 Gargiulo G, Bradford S A, Simunek J, et al. Bacteria transport and deposition under unsaturated flow conditions: The role of water content and bacteria surface hydrophobicity. Vadose Zone J, 2008, 7: 406-419

36 Jawad A, Heritage J, Snelling A, et al. Influence of relative humidity and suspending menstrua on survival of Acinetobacter spp. on dry surfaces. J Clin Microbiol, 1996, 34: 2881-2887

37 Buxton A E, Anderson R L, Werdegar D, et al. Nosocomial respiratory tract infection and colonization with Acinetobacter calcoaceticus: Epidemiologic characteristics. Am J Med, 1978, 65: 507-513

38 Lim W C. Characterization of Acinetobacter sp. Lwc1 isolated from contaminated water and its survivability under different stress condition. Master's thesis. Penang: Universiti Sains Malaysia, 2014

39 Touchon M, Cury J, Yoon E J, et al. The genomic diversification of the whole Acinetobacter genus: Origins, mechanisms, and consequences. Genome Biol Evol, 2014, 6: 2866-2882

40 Juni E. Interspecies transformation of Acinetobacter: Genetic evidence for a ubiquitous genus. J Bacteriol, 1972, 112: 917-931

41 Fan R Y, Yang X Y, Wang E H, et al. Seasonal variations of soil microbial communities of undisturbed typical black soil, northeastern China (in Chinese). Soils, 2014, (2): 285-289 [范瑞英, 杨小燕, 王恩姮, 等. 未干扰黑土土壤微生物群落特征的季节变化. 土壤, 2014, (2): 285-289]

42 Christian B W, Lind O T. Multiple carbon substrate utilization by bacteria at the sediment-water interface: Seasonal patterns in a stratified eutrophic reservoir. Hydrobiologia, 2007, 586: 43-56

43 Fierer N, Ladau J, Clemente J C, et al. Reconstructing the microbial diversity and function of pre-agricultural tallgrass prairie soils in the united states. Science, 2013, 342: 621-624

44 Freixa A, Ejarque E, Crognale S, et al. Sediment microbial communities rely on different dissolved organic matter sources along a mediterranean river continuum. Limnol Oceanogr, 2016, 61: 1389-1405

45 Cabral M P, Soares N C, Aranda J, et al. Proteomic and functional analyses reveal a unique lifestyle for Acinetobacter baumannii biofilms and a key role for histidine metabolism. J Proteome Res, 2011, 10: 3399-3417

46 Schilling D, Findeiss S, Richter A, et al. The small RNA Aar in Acinetobacter baylyi: A putative regulator of amino acid metabolism. Arch Microbiol, 2010, 192: 691-702

47 Mishra S R, Lopamudra R, Narayan P A, et al. Draft genome sequence of Acinetobacter sp. strain BMW17, a cellulolytic and plant growth-promoting bacterium isolated from the rhizospheric region of Phragmites karka of Chilika Lake, India. Genome Announc, 2016, 4: e00395-e00316

48 Al-Mutairi N Z. Variable distributional characteristics of substrate utilization patterns in activated sludge plants in Kuwait. Bioresource Technol, 2009, 100: 1524-1532

49 Dong Z Y, Hong M, Hu H J, et al. Effect of excess nitrogen loading on the metabolic potential of the bacterial community in oligotrophic coastal water (in Chinese). Acta Sci Circumst, 2018, 38: 457-466 [董志颖, 洪慢, 胡晗静, 等. 过量氮输人对塞营养海水细菌群落代 谢潜力的影响. 环境科学学报, 2018, 38: 457-466]

50 Allison S D, Martiny J B H. Resistance, resilience, and redundancy in microbial communities. Proc Natl Acad Sci USA, 2008, 105: 11512-11519

\section{补充材料}

表 S1 湖北清江和尚洞滴水样本的采集信息、理化参数和苂光有机质指数

表 S2 洞穴滴水中细菌群落结构与环境因子的圥余分析结果

本文以上补充材料见网络版 csb.scichina.com. 补充材料为作者提供的原始数据, 作者对其学术质量和内容负责. 


\title{
Seasonal variation of bacterial community and their functional diversity in drip water from a karst cave
}

\author{
Yuan Yun ${ }^{1,2}$, Xiaoyu Cheng ${ }^{1,2}$, Weiqi Wang ${ }^{1,2} \&$ Hongmei Wang ${ }^{1,2^{*}}$ \\ ${ }^{1}$ School of Environmental Studies, China University of Geosciences (Wuhan), Wuhan 430074, China; \\ ${ }^{2}$ State Key Laboratory of Biogeology and Environmental Geology, China University of Geosciences (Wuhan), Wuhan 430074, China \\ *Corresponding author, E-mail: wanghmei04@163.com, hmwang@cug.edu.cn
}

Caves are natural laboratories to study the subsurface deep biosphere, where drip water connect the caves with outside environments. Drip water may bring microorganisms and DOC from the outside environments into caves which subsequently play a fundamental role in sustaining the oligotrophic subsurface ecosystem. To understand the seasonal dynamics of bacterial communities, their potential metabolic functions, and their association with environmental factors such as precipitation, temperature, drip rate, $\mathrm{pH}, \mathrm{NO}_{3}^{-}, \mathrm{SO}_{4}^{2-}, \mathrm{Ca}^{2+}, \mathrm{Mg}^{2+}$, TOC and FI, drip water samples from Heshang Cave were collected across a two years' sampling period and subject to $16 \mathrm{~S}$ rRNA Illumina sequencing and the Biolog-ECO plates analysis.

Our results show that the richness and diversity of bacterial communities in the drip water revealed no significant seasonal difference, whereas the compositions of bacterial communities showed a clear seasonal variation, especially Acinetobacter and Pseudomonas, the dominant groups. In details, the relative abundance of Acinetobacter was higher in spring and summer seasons, while lower in autumn and winter seasons. In contrast, the relative abundance of Pseudomonas showed an opposite variation to that of Acinetobacter. Precipitation, temperature inside and outside the cave, drip rate and conductivity showed strong seasonal variations, whereas other environmental factors exhibited moderate variation within the two years' period. Among all the environmental factors investigated, precipitation significantly shaped the bacterial community structure, explaining about $27.6 \%$ of their seasonal variation. Pseudomonas significantly negatively correlated with multiple environmental factors such as precipitation, drip rate and temperature, whereas Acinetobacter showed a positive correlation only with the drip rate.

Furthermore, potential metabolic functions of bacterial communities in drip water also show clear seasonal variation as indicated both by $16 \mathrm{~S}$ rRNA PICRUSt and Biolog-ECO plate analysis. The redundancy analysis (RDA) results showed that the relative abundance of Acinetobacter demonstrated a significant relationship with the seasonal dynamics of metabolic ability. In addition, Acinetobacter revealed a remarkable positive correlation with amino acid metabolism, lipid metabolism, metabolism of terpenoids and polyketid, xenobiotics biodegradation and metabolism, and carboxylic acids metabolism. Pseudomonas showed a significant negative correlation with lipid metabolism, metabolism of terpenoids and polyketid, xenobiotics biodegradation and metabolism, and amine metabiolism.

Overall, our results showed that precipitation significantly controls the seasonal variation of bacterial communities in drip water and Acinetobacter plays an important role in the variation of bacterial functions. This the first report about the bacterial communities and their carbon utilization variation over a 2 years' period which greatly enhances our understanding about the seasonal dynamics of microbial communities and their correlation with environmental conditions.

cave drip water, bacterial community, metabolic function, environmental factor, seasonal variation

doi: 10.1360/N972018-00627 